\title{
The Assessment of Dynamic Spinal Cord Impingement by Kinematic Magnetic Resonance Imaging in Patients with Traumatic Central Cord Syndrome
}

This article was published in the following Dove Press journal:

Therapeutics and Clinical Risk Management

\begin{abstract}
Jia $\mathrm{Li}^{1,2, *}$
Da Shi ${ }^{1,2, *}$

Zijian Hua ${ }^{1,2}$

Linfeng Wang ${ }^{1,2}$

'Department of Orthopaedic Surgery, The Third Hospital of Hebei Medical University, Shijiazhuang 05005I, People's Republic of China; ${ }^{2}$ The Key Laboratory of Orthopedic Biomechanics of Hebei Province, The Third Hospital of Hebei Medical University, Shijiazhuang 05005I, People's Republic of China
\end{abstract}

*These authors contributed equally to this work
Correspondence: Linfeng Wang No. 139, Ziqiang Road, Shijiazhuang, Hebei, People's Republic of China

Tel $+86-31 \mathrm{I}-88602016$

Fax $+86-311-88602015$

Email jzwlfI899@I63.com
Background: There are few reports describing the imaging features of traumatic central cord syndrome (TCCS) in kinematic magnetic resonance imaging (kMRI) scans. The current study aimed to assess and characterize dynamic spinal cord impingement (DSCI) using kMRI and evaluate the risk factors for poor outcomes in patients with TCCS after surgery. Methods: This retrospective study included 63 patients with TCCS who underwent surgery in the authors' hospital. The American Spinal Injury Association impairment scale grade and Japanese Orthopedic Association (JOA) score were collected preoperatively and at the final follow-up to assess neurological function. Patients were divided into two groups based on the JOA score $(>$ or $<50 \%$ ), and clinical and radiographic evaluations were compared. The metrics examined included age at the time of surgery, gender, type of trauma, the interval from injury to surgery, surgical approach, pathological signs, range of motion, DSCI and the length of intramedullary hyperintensity signal (LIHS).

Results: There were statistical differences in the LIHS, DSCI and preoperative JOA score between good $(\mathrm{JOA}>50 \% ; \mathrm{n}=50)$ and poor (JOA $<50 \%, \mathrm{n}=13$ ) recovery patient groups $(\mathrm{P}<0.05)$. Logistic regression, with poor outcomes as the dependent variable, suggested independent risk associations with preoperative JOA score $(\mathrm{P}<0.05)$, DSCI $(\mathrm{P}<0.05)$ and LIHS $(\mathrm{P}<0.05)$.

Conclusion: kMRI can be effective for decision-making in the treatment of TCCS. The lower preoperative JOA score, longer high signal cord and higher dynamic spinal cord impingement were risk factors for poor outcomes in patients with TCCS after surgery.

Keywords: dynamic spinal cord impingement, traumatic central cord syndrome kinematic magnetic resonance imaging, risk factor

\section{Introduction}

Traumatic central cord syndrome (TCCS) is an incomplete spinal cord injury caused by trauma, which manifests as greater motor impairment in the upper limbs than in the lower limbs, sensory dysfunction of varying degrees below the injury level and/or bladder dysfunction. Due to preexisting degeneration of the cervical spine, conservative treatment may be not enough to restore function in TCCS cases. Therefore, surgery is often suggested to decompress the spinal cord and restore spinal stability. ${ }^{1-3}$ Magnetic resonance imaging (MRI) provides the greatest range of information and accurate delineation of soft-tissues (eg, 
intervertebral discs, spinal ligaments, and neural elements) and osseous structures (eg, facets and uncovertebral joints). This imaging modality therefore enables the detection of subtle abnormalities with high sensitivity. ${ }^{1}$ Due to the noninvasiveness or MRI, additional kinematic postures are also considered helpful, the dynamic factors of which may contribute to the pathogenesis of TCCS. Increased dynamic spinal cord impingement (DSCI) may occur during flexion or extension, when there is spinal instability, dynamic ligamentum flavum or annular bulging. ${ }^{4-6}$ Some surgeons have also reported that the degree of compression on the spinal cord is correlated with clinical symptoms. ${ }^{7,8}$ Meanwhile, others have found that patients with severe spinal cord compression can exhibit minor neural dysfunctions. However, all such studies have been conducted by comparing the degree of spinal cord compression and surgical outcomes on the basis of imaging investigations such as computed tomographic myelography and neutral position MRI. Based on these previous studies and the mechanism of injury, ${ }^{9,10}$ kinematic MRI (kMRI) may significantly aid in evaluation of DSCI at different positions for patients with TCCS. For example, Bao et al analyzed the safety of kMRI in 16 patients with spinal cord injury and found that it had greater advantages than the neutral position MRI. ${ }^{11}$ Nevertheless, as far as we know, no study has evaluated the characteristics of TCCS using kMRI and whether it can be used to identify prognostic factors of TCCS. The current study therefore aimed to assess the characteristics of DSCI by kMRI and to evaluate the risk factors for poor outcomes in patients with TCCS after surgery.

\section{Patients and Methods}

This study retrospectively reviewed the medical records of 63 patients (38 men and 25 women) with TCCS who underwent surgery in our institution between January 2015 and February 2017. The mean age at the time of surgery was $55.3 \pm 11.2$ years (range, 30-80 years) and the mean follow-up period was 16.3 months (range 12-36 months). Anterior decompression with fusion was performed in 40 patients, while the expansive open-door laminoplasty was performed in 23 patients. The inclusion criteria were as followed: history of trauma in the cervical spine; age at the time of surgery greater than 18; complying with a standard of the diagnostic criteria of TCCS; received cervical kMRI; follow-up time greater than 12 months. Exclusion criteria were as followed: structural destruction of the cervical spine (cervical vertebral body fracture, pedicle fracture or displacement, facet joint fracture or dislocation, lamina fracture); the lack of cervical spinal stenosis; trauma to the brain and limbs; the accompaniment of other diseases that can affect the evaluation of neurological function (such as motor neuron disease, Parkinson's disease, cerebral palsy and cerebral infarction, etc.). Additionally, patients were also excluded if they were unable to undergo the kMRI scans or received conservative treatment. This study was approved by the Ethics Committee of the Third Hospital of Hebei Medical University, China. The requirement for informed consent of patients was waived as all data were anonymized prior to processing and analysis. All methods were conducted in accordance with the approved guidelines.

\section{Imaging Technique and Patient Positioning}

All kMRI scans were acquired on a SIEMENS Magic View 300 System with automatic angle presentation. Patients with severe TCCS should avoid dynamic motion or superfluous loading. Therefore, after a routine supine neutral-position $\left(0^{\circ}\right)$ examination, the kinetic examination was performed with neck flexion $\left(40^{\circ}\right)$ and extension $\left(-20^{\circ}\right)$ using custom-built positioning sponges. A posterior neck receive-only surface coil was placed beneath the sponges. To ascertain the dynamic changes, the diameter of spinal cord compression in the mid-sagittal section of the head at neutral, flexed, and extended postures was evaluated on an MRI workstation with a measurement accuracy of $0.1 \mathrm{~mm}$. If the patients had multiple cord segment impediments, the most dynamically changed segment of the cord on mid-sagittal section, from flexion to extension, was utilized for analysis. The dynamic difference of cord compression was then obtained by subtracting the diameter of the compressed cord in the mid-sagittal section at flexion from that at extension.

\section{Clinical and Radiographic Evaluation}

The American Spinal Injury Association impairment scale (AIS) grade and Japanese Orthopedic Association (JOA) score were used to assess the neurological function of all patients preoperatively and at the final follow-up. The AIS grade rates patients on a scale from $\mathrm{A}$ to $\mathrm{E}$ with grade A representing complete impairment and grade E representing normal function. The recovery rate of the JOA score ([postoperative JOA score-preoperative JOA 


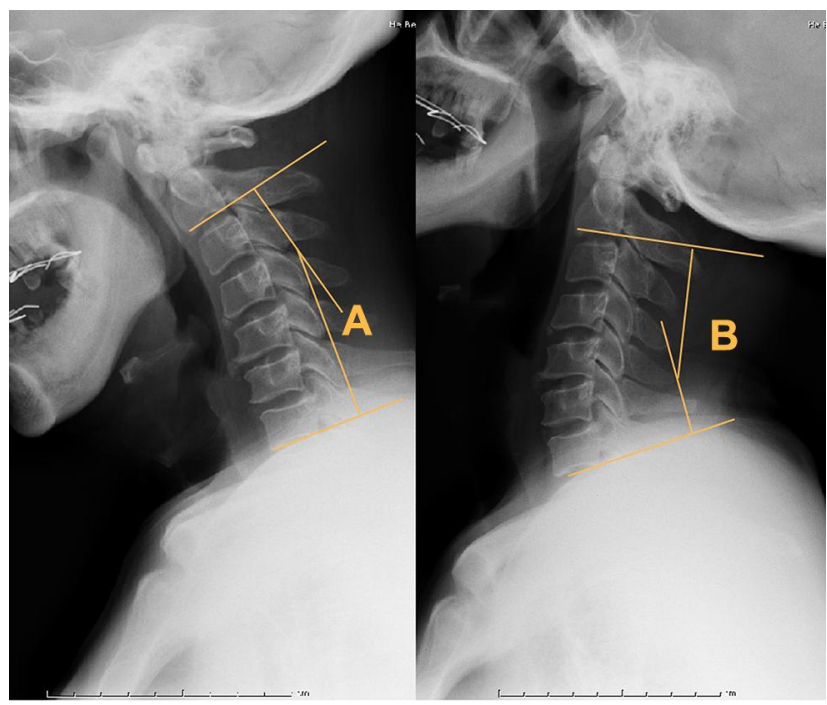

Figure I Angles created by a line parallel to the inferior aspect of the $C 2$ vertebrae and a line parallel to that of the $\mathrm{C7}$ vertebrae were measured at the flexion $(\mathbf{A})$ and extension (B) lateral views. A total ROM value was obtained via the summation of these angles.

score]/ [17-preoperative JOA score] $\times 100 \%$ ) was also calculated. Patients were then divided into a good and poor outcomes group based on whether the JOA recovery rate was $>50 \%$ or $<50 \%$, respectively. The following parameters were also obtained from all patients: age at the time of surgery, gender, type of trauma, the interval from injury to surgery, surgical approach, pathological signs, range of motion (ROM) (Figure 1), DSCI (Figure 2) and the length of intramedullary hyperintensity signal (LIHS) (Figure 3). All parameters were measured by two experienced orthopedic surgeons (Z.H. and D.S.) who were blinded to the patients' information. The average of the two measurements were used in the subsequent analyses. The intraclass correlation coefficient (ICC) was used to evaluate the reliability of the measurement. According to the ICC values, the intra-observer and inter-observer reliability of these measurements were 0.88 and 0.81 , respectively. Thus, the measurement methods used in this study were confirmed to be highly reliable.

\section{Statistical Analysis}

Statistical analyses were performed using the SPSS software (version 21.0, Chicago, IL, USA). A P value less than 0.05 was considered statistically significant. When applicable, variables are represented as the mean \pm standard deviation. Parametric statistical analysis was performed using a Student's $t$-test and an independent $t$-test or Chisquare test was used to identify significant differences between groups. Multivariate logistic regression analysis was used to identify risk factors related to poor outcomes.

\section{Results}

The primary cause of TCCS in patients was falling and traffic accidents, followed by sports injuries. No obvious surgery-related complications occurred after the operation

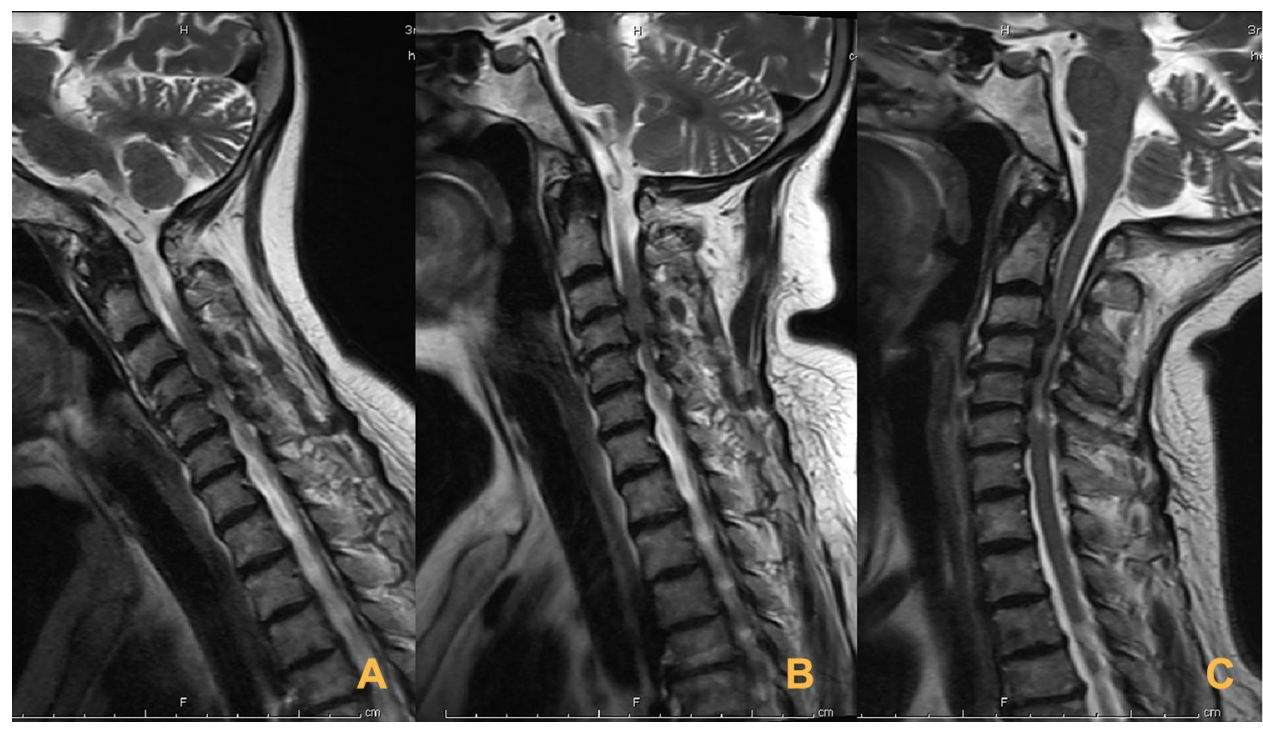

Figure 2 A 50-year-old patient with TCCS. T2-weighted MR images depict disk protrusion and hypertrophy of the ligamentum flavum at the C3-6 level. (A) In flexion, decompression of the cord impingement is seen. The diameter of the spinal cord at C3/4, C4/5, C5/6 level is $4.3 \mathrm{~mm}, 4.4 \mathrm{~mm}, 4.3 \mathrm{~mm}$, respectively. (B) In the neutral position, ventral and dorsal cord compression (pincer effect) is seen at the C3-6 level. The diameter of the spinal cord at the C3/4, C4/5, C5/6 level is $2.9 \mathrm{~mm}, 3.2 \mathrm{~mm}$, $3.5 \mathrm{~mm}$, respectively. (C) In extension, more severe spinal cord impingement is seen. The diameter of the spinal cord at the C3/4, C4/5, C5/6 level is $1.8 \mathrm{~mm}, 2.2 \mathrm{~mm}$, $2.5 \mathrm{~mm}$, respectively. The most dynamically changed segment of the spinal cord from flexion to extension was at the C3/4 level, the difference being $2.5 \mathrm{~mm}$. 


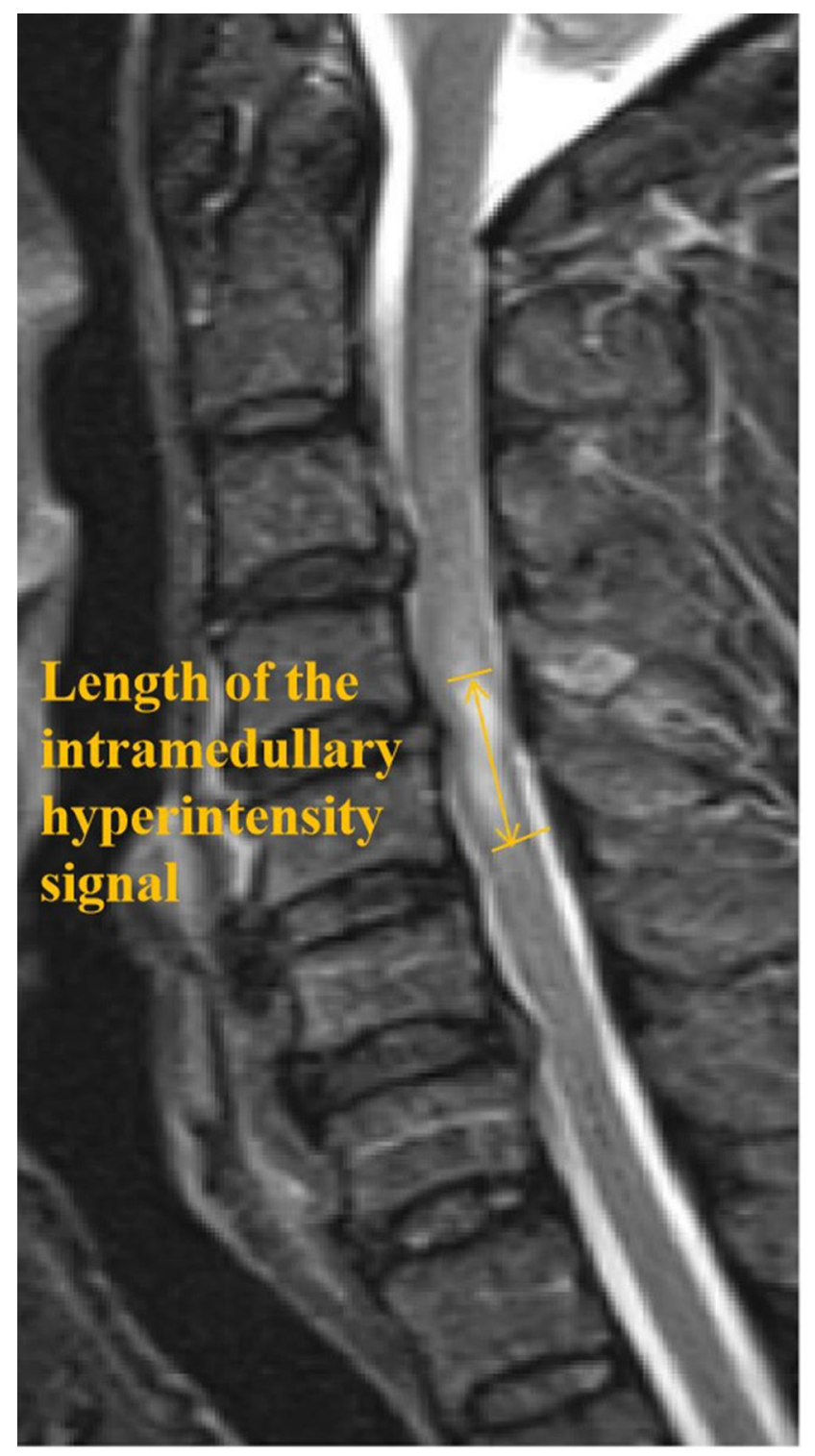

Figure 3 Length of the intramedullary hyperintensity signal (LIHS) - yellow arrow. This distance was measured as the proximal-distal range of the intramedullary hyperintensity signal. The LIHS represents the spatial range of spinal cord injury. The longer the hyperintensity-affected spinal cord, the more severe the spinal cord function is damaged after injury and the worse the prognosis becomes.

that required additional surgery. At the time of admission, 16 cases were rated as $\mathrm{C}$ and 47 cases were rated D, according to the AIS grading scale. At the last follow-up, all 16 patients with grade $\mathrm{C}$ returned to grade $\mathrm{D}$, and 15 patients with grade D returned to grade E. None of the patients' AIS grade deteriorated to a worse grade. The JOA score significantly increased from $9.9 \pm 2.8$ to 14.1 \pm 3.1 , and the recovery rate of the JOA score was $65.3 \%$.

The 63 patients were divided into two groups based on the recovery rate of JOA score, with 50 patients in the good outcomes group and 13 patients in the poor outcomes group, as shown in Table 1. No statistically significant
Table I Comparison of Demographic Data of Patients with Good Outcomes Group and Poor Outcomes Group at Admission

\begin{tabular}{|l|l|l|l|}
\hline & $\begin{array}{l}\text { Good } \\
\text { Group }\end{array}$ & $\begin{array}{l}\text { Poor } \\
\text { Group }\end{array}$ & P value \\
\hline $\begin{array}{l}\text { Age (years) } \\
\text { Gender (M/F) }\end{array}$ & $53.2 \pm 13.2$ & $58.8 \pm 10.6$ & 0.089 \\
Pathological signs & $28 / 22$ & $10 / 3$ & 0.215 \\
Positive & 23 & 8 & 0.365 \\
Negative & 27 & 5 & \\
Type of injury & & & \\
Sport injury & 5 & 1 & 0.586 \\
Falling & 26 & 5 & \\
Traffic accidents & 19 & 7 & \\
Interval from injury to & $26.3 \pm 6.2$ & $21.5 \pm 7.6$ & 0.318 \\
surgery & & & \\
Surgical approach & $47.1 \pm 5.5$ & $49.6 \pm 6.8$ & 0.522 \\
Anterior & 33 & 7 & \\
Posterior & 17 & 6 & \\
ROM & $39.6 \pm 13.3$ & $43.5 \pm 11.6$ & 0.195 \\
DSCl & $1.2 \pm 0.6$ & $2.7 \pm 1.1$ & $P<0.05$ \\
LIHS & $16.1 \pm 10.7$ & $25.9 \pm 13.9$ & $P<0.05$ \\
Preoperative JOA & $10.5 \pm 2.6$ & $8.0 \pm 3.3$ & $P<0.05$ \\
\hline
\end{tabular}

differences were found between the two groups regarding preoperative demographic factors, including gender $(\mathrm{P}=0.215)$, the interval from injury to surgery $(\mathrm{P}=0.318)$, type of injury $(\mathrm{P}=0.586)$ and surgical approach $(\mathrm{P}=0.522)$. There were, however, statistical differences in the LIHS $(\mathrm{P}<0.05)$, DSCI $(\mathrm{P}<0.05)$ and preoperative JOA score $(\mathrm{P}<0.05)$ between the two groups.

Multiple logistic regression analysis was performed to assess the relative impact of the acquired variables on poor outcomes. The results of all univariate analyses between the good and poor outcomes groups are shown in Table 2. The age, preoperative JOA score, LIHS, DSCI and ROM were utilized as dependent variables in a forward stepwise univariate analysis with a $\mathrm{p}$ value cutoff of 0.2 . This analysis indicated that the preoperative JOA score $(\mathrm{P}<0.05)$, DSCI $(\mathrm{P}<0.05)$ and LIHS $(\mathrm{P}<0.05)$ were

Table 2 Risk Factors for Traumatic Central Cord Syndrome: Multiple Logistic Regression Analysis

\begin{tabular}{|l|l|l|l|}
\hline Risk Factor & $\mathbf{P}$ & OR & $\mathbf{9 5 \%} \mathbf{~ C l}$ \\
\hline Age (years) & 0.526 & 1.969 & $0.356-10.677$ \\
ROM $\left(^{\circ}\right)$ & 0.335 & 2.635 & $1.329-5.396$ \\
LIHS $(\mathrm{mm})$ & $\mathrm{P}<0.05$ & 5.529 & $1.722-17.762$ \\
DSCl $(\mathrm{mm})$ & $\mathrm{P}<0.05$ & 3.088 & $1.553-6.632$ \\
Preoperative JOA & $\mathrm{P}<0.05$ & 2.689 & $1.329-5.439$ \\
\hline
\end{tabular}


significant risk factors associated with poor outcomes after surgery.

\section{Discussion}

It is believed that surgical treatment is a reasonable option for TCCS, especially for patients with cervical spinal stenosis and progressive aggravation of clinical symptoms. ${ }^{12,13}$ Surgical treatments are becoming more popular for the treatment of TCCS as the mortality rate of these patients has been shown to be significantly lower than that of non-surgical patients. ${ }^{14}$ The main mechanism of injury for TCCS is reported as cervical spine hyperextension which causes acute spinal stenosis. This type of injury also often causes anterior herniated disc tissue or osteophyte and posterior ligamentum flavum "clamping" of the cervical spinal cord. ${ }^{3,15,16}$ Some patients show obvious clinical symptoms with mild spinal cord compression; however, on the contrary, some patients with severe spinal cord compression exhibit little neural dysfunction. The kMRI provides information about dynamic changes in the hypotonic ligamentum flavum and annular bulging, and most importantly reveals the impact of these changes on the spinal cord. ${ }^{6,17,18}$ Surgical interventions to relieve spinal cord compression can reduce spinal cord edema, improve spinal cord blood supply, create favorable conditions for the recovery of spinal cord function, and prevent delayed neurological deterioration. The 63 TCCS patients included in this study were treated with surgery and no surgery-related complications occurred after operation. The AIS grade and JOA score at the last follow-up were higher than those at admission, suggesting that the surgical treatment was safe and able to effectively restore the patients' spinal cord function.

At present, factors related to the prognosis of TCCS and reported in literature include the admission AIS grade, central spinal canal sagittal diameter, MRI T2 image spinal cord hyperintensity length, number of spinal stenosis segments, timing of surgery, age and surgical approach. ${ }^{19,20}$ Some works have reported that increased cervical cord impingement is seen in $27 \%$ to $31 \%$ of extension and in $3-5 \%$ of flexion postures. ${ }^{21,22}$ In the study by Chen et al, more patients exhibited reduced spinal stenosis with spinal cord decompression at flexion compared to that at extension. ${ }^{21}$ These results, as well as those from other studies, indicate a minor role of flexion to reducing functional spinal cord impingement. ${ }^{18}$ Nevertheless, these previous studies mainly focused on evaluating the dynamic changes in spinal canal stenosis during neck flexion and extension in patients with cervical degenerative disease. By contrast, in the current work, we studied the degree of dynamic cord impingement in patients with TCCS.

In the present study, the most severe spinal cord compression was found at extension across all three positions examined. From extension to neutral to flexion, the degree of spinal cord compression gradually decreased. There was no further aggravated spinal cord impingement at flexion compared to neutral and extension in all patients. At extension, due to the backward gliding of the vertebrae, protrusion of discs and buckling of the posterior longitudinal ligaments can lead to narrowing of the anterior subarachnoid space and anterior cervical cord compression. However, because of the physiological shortening of the cervical canal with dorsal shifting of the cervical cord, dura matter folding, spinal cord thickening, ligamenta flava buckling and posterior spinal stenosis increases. ${ }^{21,23,24}$ This was likely why we saw a dramatic increase in the incidence of aggravated spinal cord impingement at extension. Recently, Kato et al reported that following the application of $5^{\circ}-10^{\circ}$ of flexion on a threedimensional finite element model, spinal cord stress increased and manifested as observable effects in the gray matter, posterior funiculus, and a portion of the lateral funiculus on the spinal cord. ${ }^{25}$ Furthermore, the stress distribution of the spinal cord under static compression increased with faster flexion speed of the spinal cord. These results showed that although the degree of cord compression reduced gradually and even thoroughly decompress at flexion, there was still additional stress on the spinal cord.

In the poor outcomes group, there tended to be a rapid spinal cord impingement during trauma. Based on the present results, we speculate that with a higher degree of dynamic impingement, the spinal cord can hardly gain sufficient space and time to adapt to the stimulation of rapid trauma. Consequently, the spinal cord exhibits rapid development functional decompensation and neurological function should recover soon afterward. This process coincides with the improvement in postoperative JOA score in good outcomes patients in our study who received a surgical intervention early. However, if dynamic cord stimulation persistently existed, it can cause severe myelopathy and even irreversible spinal cord lesions. In the good outcomes group, the lower degree of dynamic cord stimulation led us to believe that the spinal cord can gain partial space and time to adapt to the stimulation/trauma until decompensation can be achieved. Therefore, it is likely 
that dynamic cord stimulation is important to the pathogenesis and development of TCCS.

The LIHS represents the spatial range of spinal cord injury. The longer the hyperintensity-affected spinal cord, the more severe the spinal cord function is damaged after injury and the worse the prognosis becomes. Considering the difference of LIHS in two groups, we speculated that a relatively short LIHS may be a main factor leading to the acceptable surgical outcome in the good outcomes group. Therefore, this result also demonstrates that the short LIHS contributes to reducing the lesion of myelopathy from trauma.

On the other hand, we considered that dynamic cervical cord stimulation is important to the pathogenesis and development of cervical compressive myelopathy. A short duration of cervical cord stimulation would contribute to reducing the lesion of myelopathy from repeated minor traumas. Long-term high dynamic cord impingements are more dangerous than relative static spinal cord compressions for patients with cervical compressive myelopathy, and is also an important factor for the poor outcomes after surgery. However, due to insufficient clinical and radiographic data on the daily life of patients, further research should be conducted to verify these findings.

In our study, the ROM of the cervical spine and the degree of dynamic cord impingement in the good outcomes group were lower than those in the poor outcomes group. The JOA score of patients in the poor outcomes group was inferior to that in the good outcomes group. Masaki et $\mathrm{al}^{26}$ recently suggested that hypermobility of vertebrae at the spinal cord compression level is a risk factor for poor surgical outcomes after laminoplasty. In support of this argument, the recovery rates between the two groups in the present study showed a significant difference at the final follow up. Furthermore, the degree of the ROM in the poor outcomes group was a little higher than that in the good outcomes group. However, after the multiple logistic regression analysis, the ROM was not a risk factor of poor clinical outcomes. On the other hand, the paravertebral soft tissue of the cervical spine injury might compromise the whole ROM.

There are several limitations in the present study that should be noted. First, the small sample size and retrospective nature make it difficult to sample all types of TCCS and exclude the possibility of selection bias. Second, owing to the cost of kMRI and the concomitant complications, some patients could not endure the different positions employed in this work for a long time. In addition, electrophysiological monitoring is necessary to be performed in future examinations as complimentary measurements. Finally, a long-term prospective study should be performed at a large scale to demonstrate the correlation between the DSCI and LIHS, and their impact on the clinical outcomes of TCCS.

\section{Conclusion}

We conclude that kMRI is an effective approach for decision-making in the treatment of TCCS. The lower preoperative JOA score, longer high signal cord and higher dynamic spinal cord impingement are risk factors for poor outcomes in patients with TCCS after surgery.

\section{Abbreviations}

TCCS, traumatic central cord syndrome; kMRI, kinematic magnetic resonance imaging; AIS, American Spinal Injury Association impairment scale; JOA, Japanese Orthopedic Association; DSCI, dynamic spinal cord impingement; LIHS, length of intramedullary hyperintensity signal; $\mathrm{ROM}$, range of motion.

\section{Ethics Approval and Consent to Participate}

The Ethics Committee of Third Hospital of Hebei Medical University approved this study. Patient consent for review of medical records was not required, as all data were de-identified. All protocols were conducted in accordance with the research principles in the Declaration of Helsinki.

\section{Funding}

There was no direct funding source aligned to this study.

\section{Disclosure}

This material has not been published and is not under consideration elsewhere. The authors declare that they have no competing interests.

\section{References}

1. Yelamarthy PKK, Chhabra HS, Vaccaro A, et al. Management and prognosis of acute traumatic cervical central cord syndrome: systematic review and Spinal Cord Society-Spine Trauma Study Group position statement. Eur Spine J. 2019;28(10):2390-2407. doi:10.1007/ s00586-019-06085-z

2. Anderson KK, Tetreault L, Shamji MF, et al. Optimal timing of surgical decompression for acute traumatic central cord syndrome: a systematic review of the literature. Neurosurgery. 2015;77(Suppl 4):S15-S32. doi:10.1227/NEU.0000000000000946 
3. Khorasanizadeh M, Yousefifard M, Eskian M. et al. Neurological recovery following traumatic spinal cord injury: a systematic review and meta-analysis. J Neurosurg Spine. 2019:1-17. doi:10.3171/ 2018.10.SPINE18802

4. Moroz J, Reinsberg SA. Dynamic contrast-enhanced MRI. Methods Mol Biol. 2018;1718:71-87.

5. Paholpak P, Tamai K, Shoell K, et al. Can multi-positional magnetic resonance imaging be used to evaluate angular parameters in cervical spine? A comparison of multi-positional MRI to dynamic plain radiograph. Eur Spine J. 2018;27(5):1021-1027. doi:10.1007/ s00586-017-5306-1

6. Yecies D, Fogel N, Edwards M, et al. Safety of dynamic magnetic resonance imaging of the cervical spine in children performed without neurosurgical supervision. World Neurosurg. 2018;116:e1188e1193. doi:10.1016/j.wneu.2018.05.210

7. Kadanka Z, Kerkovsky M, Bednarik J, et al. Cross-sectional transverse area and hyperintensities on magnetic resonance imaging in relation to the clinical picture in cervical spondylotic myelopathy. Spine. 2007;32(23):2573-2577. doi:10.1097/BRS.0b013e318158cda0

8. Yamazaki T, Yanaka K, Sato H, et al. Cervical spondylotic myelopathy: surgical results and factors affecting outcome with special reference to age differences. Neurosurgery. 2003;52(1):122-126. doi:10.1097/00006123-200301000-00015

9. Bednarik J, Kadanka Z, Dusek L, et al. Presymptomatic spondylotic cervical cord compression. Spine. 2004;29(20):2260-2269. doi:10.1097/01.brs.0000142434.02579.84

10. Xiong C, Daubs MD, Scott TP, et al. Dynamic evaluation of the cervical spine and the spinal cord of symptomatic patients using a kinetic magnetic resonance imaging technique. Clin Spine Surg. 2017;30(8):E1149-E1155. doi:10.1097/BSD.0000000000000429

11. Bao Y, Zhong X, Zhu W, et al. Feasibility and safety of cervical kinematic magnetic resonance imaging in patients with cervical spinal cord injury without fracture and dislocation. Orthop Surg. 2020;12(2):570-581. doi:10.1111/os.12663

12. Kato S, Fehlings MG. Treatment of central cord syndrome without associated spinal instability. Semin Spine Surg. 2017;29(1):60-64. doi:10.1053/j.semss.2016.09.010

13. Fehlings MG, Martin AR, Tetreault LA, et al. A clinical practice guideline for the management of patients with acute spinal cord injury: recommendations on the role of baseline magnetic resonance imaging in clinical decision making and outcome prediction. Global Spine J. 2017;7(3 Suppl):221S-230S. doi:10.1177/ 2192568217703089

14. Yoshihara H, Yoneoka D. Trends in the treatment for traumatic central cord syndrome without bone injury in the United States from 2000 to 2009. J Trauma Acute Care Surg. 2013;75 (3):453-458. doi:10.1097/TA.0b013e31829cfd7f
15. Stevenson CM, Dargan DP, Warnock J, et al. Traumatic central cord syndrome: neurological and functional outcome at 3 years. Spinal Cord. 2016;54(11):1010-1015. doi:10.1038/sc.2016.34

16. Li XF, Dai LY. Acute central cord syndrome: injury mechanisms and stress features. Spine. 2010;35(19):E955-64. doi:10.1097/ BRS.0b013e3181c94cb8

17. Dalbayrak S, Yaman O, Firidin MN, et al. The contribution of cervical dynamic magnetic resonance imaging to the surgical treatment of cervical spondylotic myelopathy. Turk Neurosurg. 2015;25 (1):36-42. doi:10.5137/1019-5149.JTN.9082-13.1

18. Tykocki T, Du Plessis J, Wynne-Jones G. Analysis of morphometric parameters in cervical canal stenosis on neutral and dynamic magnetic resonance imaging. World Neurosurg. 2018;114:e317-e322. doi:10.1016/j.wneu.2018.02.179

19. Thompson C, Gonsalves JF, Welsh D. Hyperextension injury of the cervical spine with central cord syndrome. Eur Spine J. 2015;24 (1):195-202. doi:10.1007/s00586-014-3432-6

20. Kepler CK, Kong C, Schroeder GD, et al. Early outcome and predictors of early outcome in patients treated surgically for central cord syndrome. J Neurosurg Spine. 2015;23(4):490-494. doi:10.3171/ 2015.1.SPINE141013

21. Chen CJ, Hsu HL, Niu CC, et al. Cervical degenerative disease at flexion-extension MR imaging: prediction criteria. Radiology. 2003;227(1):136-142. doi:10.1148/radiol.2271020116

22. Baron EM, Young WF. Cervical spondylotic myelopathy: a brief review of its pathophysiology, clinical course, and diagnosis. Neurosurgery. 2007;60(1Supp1 1):S35-S41. doi:10.1227/01. NEU.0000215383.64386.82

23. Zeng C, Xiong J, Wang JC, et al. The evaluation and observation of "Hidden" hypertrophy of cervical ligamentum flavum, cervical canal, and related factors using kinetic magnetic resonance imaging. Global Spine J. 2016;6(2):155-163. doi:10.1055/s-0035-1557140

24. Kuwazawa Y, Bashir W, Pope MH, et al. Biomechanical aspects of the cervical cord: effects of postural changes in healthy volunteers using positional magnetic resonance imaging. J Spinal Disord Tech. 2006;19(5):348-352. doi:10.1097/01.bsd.0000203273.90004.eb

25. Kato Y, Kanchiku T, Imajo Y, et al. Flexion model simulating spinal cord injury without radiographic abnormality in patients with ossification of the longitudinal ligament: the influence of flexion speed on the cervical spine. J Spinal Cord Med. 2009;32(5):555-559. doi:10.1080/10790268.2009.11754557

26. Masaki Y, Yamazaki M, Okawa A, et al. An analysis of factors causing poor surgical outcome in patients with cervical myelopathy due to ossification of the posterior longitudinal ligament: anterior decompression with spinal fusion versus laminoplasty. J Spinal Disord Tech. 2007;20(1):7-13. doi:10.1097/01. bsd.0000211260.28497.35
Therapeutics and Clinical Risk Management

\section{Publish your work in this journal}

Therapeutics and Clinical Risk Management is an international, peerreviewed journal of clinical therapeutics and risk management, focusing on concise rapid reporting of clinical studies in all therapeutic areas, outcomes, safety, and programs for the effective, safe, and sustained use of medicines. This journal is indexed on PubMed Central, CAS,
EMBase, Scopus and the Elsevier Bibliographic databases. The manuscript management system is completely online and includes a very quick and fair peer-review system, which is all easy to use. Visit http://www.dovepress.com/testimonials.php to read real quotes from published authors. 